ANNALES

POLONICI MATHEMATICI

LXXVI.3 (2001)

\title{
On locally bounded solutions of Schilling's problem
}

\author{
by Janusz Morawiec (Katowice)
}

\begin{abstract}
We prove that for some parameters $q \in(0,1)$ every solution $f: \mathbb{R} \rightarrow \mathbb{R}$ of the functional equation$$
f(q x)=\frac{1}{4 q}[f(x-1)+f(x+1)+2 f(x)]
$$

which vanishes outside the interval $[-q /(1-q), q /(1-q)]$ and is bounded in a neighbourhood of a point of that interval vanishes everywhere.
\end{abstract}

Introduction. Considering a physical problem R. Schilling [18] came to the functional equation

$$
f(q x)=\frac{1}{4 q}[f(x-1)+f(x+1)+2 f(x)]
$$

where $q \in(0,1)$ is a fixed number, and to its solutions $f: \mathbb{R} \rightarrow \mathbb{R}$ satisfying the boundary condition

$$
f(x)=0 \quad \text { for }|x|>Q
$$

where

$$
Q=\frac{q}{1-q}
$$

The physical background of this problem can also be found in [9] by G. Derfel and R. Schilling and in [11] by R. Girgensohn.

In what follows any solution $f: \mathbb{R} \rightarrow \mathbb{R}$ of (1) satisfying (2) will be called a solution of Schilling's problem.

The first nontrivial continuous solution of Schilling's problem was given by $\mathrm{R}$. Schilling himself for $q=1 / 2$. This solution is defined by

$$
f_{1}(x)=\max \{1-|x|, 0\} \quad \text { for } x \in \mathbb{R} .
$$

2000 Mathematics Subject Classification: 39B12, 39B22.

Key words and phrases: Schilling's problem, bounded solution. 
K. Baron, A. Simon and P. Volkmann [3] showed that if $n$ is a positive integer and $q=1 / \sqrt[n]{2}$, then the convolution

$$
f_{1}(x) \star f_{1}(q x) \star \ldots \star f_{1}\left(q^{n-1} x\right)
$$

is a nontrivial continuous solution of Schilling's problem. They also proved that if $q \in(0,1 / 2)$ and $f$ is a nontrivial Lebesgue integrable solution of Schilling's problem, then

$$
\int_{0}^{\varepsilon}|f(x)|^{(\log q) / \log (2 q)} d x=+\infty
$$

for every $\varepsilon>0$. In particular, for every $q \in(0,1 / 2)$ every bounded Lebesgue measurable solution of Schilling's problem vanishes almost everywhere. (Note that in [3] by K. Baron, A. Simon and P. Volkmann and in [19] by A. Simon and P. Volkmann distributional solutions of Schilling's problem are considered.) The case $q \in(1 / 2,1)$ is quite different. Namely, from the paper [9] by G. Derfel and R. Schilling it follows that for almost all $q \in(1 / 2,1)$ there are nontrivial continuous solutions. However, if the inverse of $q$ is a Salem number [6], then such solutions do not exist (cf. also [15] where nonexistence of nontrivial continuous solutions of Schilling's problem was proved for the golden ratio $q=(\sqrt{5}-1) / 2)$.

K. Baron and P. Volkmann [4] (see also [8] by I. Daubechies and J. C. Lagarias) proved that for every $q \in(0,1)$ the vector space of Lebesgue integrable solutions of Schilling's problem is at most one-dimensional. (The same concerns Riemann integrable solutions; see [10] by W. Förg-Rob.) It is known that the vector space of Lebesgue integrable solutions of Schilling's problem is zero-dimensional for $q \in(0,1 /(2 \sqrt{2})$ ) (see [16] by Y. Peres and B. Solomyak) and also for those $q \neq 1 / 2$ for which the inverse of $q$ is a Pisot number (see [7] by J. M. Borwein and R. Girgensohn). However, it is one-dimensional for almost all $q \in(1 /(2 \sqrt{2}), 1)$ (see [16] by Y. Peres and B. Solomyak). Up to now the only explicitly given $q$ 's for which the vector space of integrable solutions is one-dimensional are $1 / \sqrt[n]{2}$ given by K. Baron, A. Simon and P. Volkmann [3]. If the vector space of Lebesgue integrable solutions of Schilling's problem is one-dimensional, then every nonzero function from this space is either positive or negative (almost everywhere) on its support (see [12]) and according to [5] by L. Bartłomiejczyk, Schilling's problem has also strange solutions; e.g. such that their graph meets every Borel subset of $[-Q, Q] \times \mathbb{R}$ with uncountable vertical projection.

Bounded solutions interesting from the physical point of view were first examined by K. Baron [5]. His result says that for $q \in(0, \sqrt{2}-1]$ the zero function is the only solution of Schilling's problem which is bounded in a neighbourhood of the origin. Generalizations of this result can be found in [14] and [13] where it is proved among other things that for $q \leq 1 / 3$ the 
zero function is the only solution of Schilling's problem which is bounded in a neighbourhood of a point of the set

$$
\left\{\varepsilon \sum_{i=1}^{n} q^{i}: n \in \mathbb{N} \cup\{0,+\infty\}, \varepsilon \in\{-1,1\}\right\}
$$

and no point outside (3) has this property. Note that for $q=1 / 3$ the set (3) coincides with the interval $[-Q, Q]$.

More details on Schilling's problem can be found in [11] by R. Girgensohn, in [2, Section 5] by K. Baron and W. Jarczyk and in [17].

In the present paper we are interested in finding parameters $q \in(1 / 3,1 / 2)$ for which the zero function is the only solution of Schilling's problem which is bounded in a neighbourhood of a point of $[-Q, Q]$. We make the following definition.

Definition. Let $x \in[-Q, Q]$.

We say $x \in B_{q}$ if the zero function is the only solution of Schilling's problem which is bounded in a neighbourhood of $x$.

We say $x \in C_{q}$ if the zero function is the only solution of Schilling's problem which is continuous at $x$.

We say $x \in Z_{q}$ if the zero function is the only solution of Schilling's problem which vanishes in a neighbourhood of $x$.

It is easily seen that

$$
B_{q} \subset C_{q} \subset Z_{q} \subset[-Q, Q]
$$

for every $q \in(0,1)$.

Main results. For the convenience of the reader we repeat four relevant facts from [13] without proofs.

REMARK 1. Assume $f$ is a solution of Schilling's problem. If $q \neq 1 / 4$, then $f(-Q)=f(Q)=0$. If $q<1 / 2$, then $f(0)=0$.

REMARK 2. If $f$ is a solution of Schilling's problem, then so is the function $g: \mathbb{R} \rightarrow \mathbb{R}$ defined by $g(x)=f(-x)$.

LEMMA 1. Assume $q \in(0,1 / 2)$. If a solution of Schilling's problem vanishes either on $(-q, 0)$ or on $(0, q)$, then it vanishes everywhere.

LEMMA 2. Assume $q \in(0,1 / 2)$. If $f$ is a solution of Schilling's problem, then

$$
f\left(q^{N+M} x+\varepsilon \sum_{m=1}^{M} q^{m}\right)=\left(\frac{1}{2}\right)^{M}\left(\frac{1}{2 q}\right)^{N+M} f(x)
$$

for every $x \in(Q-1,1-Q)$ (for every $x \in[Q-1,1-Q]$ if $q \neq 1 / 4)$, every $\varepsilon \in\{-1,1\}$, and any nonnegative integers $M$ and $N$. 
We first deal with the number $q=(3-\sqrt{5}) / 2$.

Lemma 3. Let $q=(3-\sqrt{5}) / 2$. If $f$ is a solution of Schilling's problem, then

$$
f\left(q^{N} x-q^{N}\right)=\frac{1}{2}\left(\frac{1}{2 q}\right)^{N} f(x)
$$

for every $x \in[0,1-Q]$ and every positive integer $N$.

Proof. Observe that $q \in(1 / 3,1 / 2)$,

$$
q^{2}-3 q+1=0 \text { and } Q=1-q .
$$

Fix $x_{0} \in[0,1-Q]$ and put $x=x_{0}-1$. Since $x-1<x \leq-Q$, by $(1)$, (2) and Remark 1 we have

$$
f\left(q x_{0}-q\right)=f(q x)=\frac{1}{4 q}[f(x-1)+f(x+1)+2 f(x)]=\frac{1}{4 q} f\left(x_{0}\right) .
$$

Fix now a positive integer $N$ and assume that (4) holds for every $x \in$ $[0,1-Q]$. Fixing $x_{0} \in[0,1-Q]$ and putting $x=q^{N} x_{0}-q^{N}$ we see that $x-1<-1<-Q$ and $x+1 \geq-q+1=Q$. Consequently,

$$
\begin{aligned}
f\left(q^{N+1} x_{0}-q^{N+1}\right) & =\frac{1}{4 q}[f(x-1)+f(x+1)+2 f(x)] \\
& =\frac{1}{2 q} f(x)=\frac{1}{2 q} f\left(q^{N} x_{0}-q^{N}\right)=\frac{1}{2}\left(\frac{1}{2 q}\right)^{N+1} f\left(x_{0}\right) .
\end{aligned}
$$

Lemma 4. Let $q=(3-\sqrt{5}) / 2$. If $f$ is a solution of Schilling's problem, then for any nonnegative integers $k$ and $l$ satisfying

$$
|k-l q|<Q
$$

there exist a positive real $\alpha_{k, l}$ and a positive integer $n_{k, l}$ such that

$$
\begin{aligned}
f\left(q^{n} x+k-l q\right) & =\alpha_{k, l}\left(\frac{1}{2 q}\right)^{n} f(x), \\
f\left(q^{n} x-q^{n}-k+l q\right) & =\frac{1}{2} \alpha_{k, l}\left(\frac{1}{2 q}\right)^{n} f(x),
\end{aligned}
$$

for every integer $n \geq n_{k, l}$ and every $x \in[0,1-Q]$.

Proof. With the help of (5) we check at once that if nonnegative integers $k$ and $l \leq 4$ satisfy $(6)$, then

$$
(k, l) \in\{(0,0),(0,1),(1,2),(1,3),(1,4),(2,4)\} .
$$

Put

$$
\begin{gathered}
\alpha_{0,0}=1, \quad \alpha_{0,1}=\alpha_{1,3}=1 / 2, \quad \alpha_{1,2}=\alpha_{1,4}=1 / 4, \quad \alpha_{2,4}=1 / 8 \\
n_{0,0}=n_{0,1}=n_{1,2}=n_{1,3}=n_{1,4}=n_{2,4}=4
\end{gathered}
$$


If $x \in[0,1-Q]$, then using Lemma 2, (5), (1), (2), Remark 1 and Lemma 3 we find that for every integer $n \geq 2$ the following equalities hold:

$$
\begin{aligned}
f\left(q^{n} x\right)= & \left(\frac{1}{2 q}\right)^{n} f(x)=\alpha_{0,0}\left(\frac{1}{2 q}\right)^{n} f(x), \\
f\left(q^{n} x-q\right)= & \frac{1}{2}\left(\frac{1}{2 q}\right)^{n} f(x)=\alpha_{0,1}\left(\frac{1}{2 q}\right)^{n} f(x), \\
f\left(q^{n} x+1-2 q\right)= & f\left(q^{n} x-q^{2}+q\right) \\
= & \frac{1}{4 q}\left[f\left(q^{n-1} x-q\right)+f\left(q^{n-1} x-q+2\right)\right. \\
& \left.+2 f\left(q^{n-1} x-q+1\right)\right] \\
= & \frac{1}{4 q} f\left(q^{n-1} x-q\right) \\
= & \frac{1}{4 q} \frac{1}{2}\left(\frac{1}{2 q}\right)^{n-1} f(x) \\
= & \alpha_{1,2}\left(\frac{1}{2 q}\right)^{n} f(x), \\
= & f\left(q^{2}\left(q^{n-2} x\right)-q^{2}\right)=\frac{1}{2}\left(\frac{1}{2 q}\right)^{2} f\left(q^{n-2} x\right) \\
= & \frac{1}{2}\left(\frac{1}{2 q}\right)^{2}\left(\frac{1}{2 q}\right)^{n-2} f(x) \\
= & \alpha_{1,3}\left(\frac{1}{2 q}\right)^{n} f(x), \\
= & f\left(q^{n} x+1-q^{2}-q\right)=\left(\frac{1}{2}\right)^{2}\left(\frac{1}{2 q}\right)^{n} f(x) \\
= & \alpha_{1,4}\left(\frac{1}{2 q}\right)^{n} f(x), \\
f\left(q^{n} x+1-4 q\right) &
\end{aligned}
$$

and if $n \geq 3$, then using also the third of the above equalities we get

$$
\begin{aligned}
f\left(q^{n} x+2-4 q\right)= & f\left(q^{n} x-2 q^{2}+2 q\right) \\
= & \frac{1}{4 q}\left[f\left(q^{n-1} x-2 q+1\right)\right. \\
& \left.+f\left(q^{n-1} x-2 q+3\right)+2 f\left(q^{n-1} x-2 q+2\right)\right] \\
= & \frac{1}{4 q} f\left(q^{n-1} x+1-2 q\right) \\
= & \frac{1}{4 q} \alpha_{1,2}\left(\frac{1}{2 q}\right)^{n-1} f(x) \\
= & \alpha_{2,4}\left(\frac{1}{2 q}\right)^{n} f(x)
\end{aligned}
$$


similarly we obtain equalities which correspond to (8):

$$
\begin{aligned}
& f\left(q^{n} x-q^{n}\right)=\frac{1}{2}\left(\frac{1}{2 q}\right)^{n} f(x)=\frac{1}{2} \alpha_{0,0}\left(\frac{1}{2 q}\right)^{n} f(x), \\
& f\left(q^{n} x-q^{n}+q\right)=\frac{1}{4 q}\left[f\left(q^{n-1} x-q^{n-1}\right)\right. \\
& \left.+f\left(q^{n-1} x-q^{n-1}+2\right)+2 f\left(q^{n-1} x-q^{n-1}+1\right)\right] \\
& =\frac{1}{4 q} f\left(q^{n-1} x-q^{n-1}\right)=\frac{1}{4 q} \frac{1}{2}\left(\frac{1}{2 q}\right)^{n-1} f(x) \\
& =\frac{1}{2} \alpha_{0,1}\left(\frac{1}{2 q}\right)^{n} f(x), \\
& f\left(q^{n} x-q^{n}-1+2 q\right)=f\left(q^{n} x-q^{n}+q^{2}-q\right) \\
& =\frac{1}{4 q}\left[f\left(q^{n-1} x-q^{n-1}+q-2\right)\right. \\
& +f\left(q^{n-1} x-q^{n-1}+q\right) \\
& \left.+2 f\left(q^{n-1} x-q^{n-1}+q-1\right)\right] \\
& =\frac{1}{4 q} f\left(q^{n-1} x-q^{n-1}+q\right) \\
& =\frac{1}{4 q} \frac{1}{2} \alpha_{0,1}\left(\frac{1}{2 q}\right)^{n-1} f(x) \\
& =\frac{1}{2} \alpha_{1,2}\left(\frac{1}{2 q}\right)^{n} f(x) \text {, } \\
& f\left(q^{n} x-q^{n}-1+3 q\right)=f\left(q^{n} x-q^{n}+q^{2}\right) \\
& =\frac{1}{4 q}\left[f\left(q^{n-1} x-q^{n-1}+q-1\right)\right. \\
& +f\left(q^{n-1} x-q^{n-1}+q+1\right) \\
& \left.+2 f\left(q^{n-1} x-q^{n-1}+q\right)\right] \\
& =\frac{1}{2 q} f\left(q^{n-1} x-q^{n-1}+q\right) \\
& =\frac{1}{2 q} \frac{1}{2} \alpha_{0,1}\left(\frac{1}{2 q}\right)^{n-1} f(x) \\
& =\frac{1}{2} \alpha_{1,3}\left(\frac{1}{2 q}\right)^{n} f(x) \text {, }
\end{aligned}
$$




$$
\begin{aligned}
& f\left(q^{n} x-q^{n}-1+4 q\right)=f\left(q^{n} x-q^{n}+q^{2}+q\right) \\
& =\frac{1}{4 q}\left[f\left(q^{n-1} x-q^{n-1}+q\right)\right. \\
& +f\left(q^{n-1} x-q^{n-1}+q+2\right) \\
& \left.+2 f\left(q^{n-1} x-q^{n-1}+q+1\right)\right] \\
& =\frac{1}{4 q} f\left(q^{n-1} x-q^{n-1}+q\right) \\
& =\frac{1}{4 q} \frac{1}{2} \alpha_{0,1}\left(\frac{1}{2 q}\right)^{n-1} f(x) \\
& =\frac{1}{2} \alpha_{1,4}\left(\frac{1}{2 q}\right)^{n} f(x), \\
& f\left(q^{n} x-q^{n}-2+4 q\right)=f\left(q^{n} x-q^{n}+2 q^{2}-2 q\right) \\
& =\frac{1}{4 q}\left[f\left(q^{n-1} x-q^{n-1}+2 q-3\right)\right. \\
& +f\left(q^{n-1} x-q^{n-1}+2 q-1\right) \\
& \left.+2 f\left(q^{n-1} x-q^{n-1}+2 q-2\right)\right] \\
& =\frac{1}{4 q} f\left(q^{n-1} x-q^{n-1}+2 q-1\right) \\
& =\frac{1}{4 q} \frac{1}{2} \alpha_{1,2}\left(\frac{1}{2 q}\right)^{n-1} f(x) \\
& =\frac{1}{2} \alpha_{2,4}\left(\frac{1}{2 q}\right)^{n} f(x) \text {. }
\end{aligned}
$$

Fix now a nonnegative integer $L \geq 5$ and assume that for any nonnegative integers $k$ and $l<L$ satisfying (6) there exist a positive real $\alpha_{k, l}$ and a positive integer $n_{k, l}$ such that (7) and (8) hold for every integer $n \geq n_{k, l}$ and every $x \in[0,1-Q]$. Let $k$ be a nonnegative integer such that

$$
|k-L q|<Q .
$$

Then

$$
1<k<L
$$

Putting

$$
y=\frac{k-L q}{q}
$$


and using (5) we see that $|y|<Q / q=Q+1$ and

$$
y=\frac{\left(3 q-q^{2}\right) k-L q}{q}=3 k-L-k q .
$$

Applying (5) again we see that $y$ belongs to one of the intervals

$$
(-Q-1,-Q),(-Q, Q-1),(Q-1,1-Q),(1-Q, Q),(Q, Q+1) .
$$

It follows that there exists a positive integer $N$ such that for every integer $n \geq N$ and $x \in[0,1-Q]$ the number $y$ belongs to one of the intervals $(10)$ together with the numbers

$$
y+q^{n-1} x, \quad y-q^{n-1} x+q^{n-1} .
$$

Moreover, making also use of (9) we have: if $y>-Q-1$, then

$$
3 k-L+1=y+k q+1>-Q+k q=-1+(k+1) q \geq-1+3 q>0 ;
$$

if $y>-Q$, then

$$
3 k-L=y+k q>-Q+k q>0
$$

and if $y>1-Q$, then

$$
3 k-L-1=y+k q-1>-Q+k q>0 .
$$

This allows us to define $\alpha_{k, L}$ and $n_{k, L}$ by

$$
\begin{array}{r}
\alpha_{k, L}= \begin{cases}\frac{1}{2} \alpha_{3 k-L+1, k} & \text { if }-Q-1<y<-Q, \\
\frac{1}{2}\left[\alpha_{3 k-L+1, k}+2 \alpha_{3 k-L, k}\right] & \text { if }-Q<y<Q-1, \\
\alpha_{3 k-L, k} & \text { if } Q-1<y<1-Q, \\
\frac{1}{2}\left[\alpha_{3 k-L-1, k}+2 \alpha_{3 k-L, k}\right] & \text { if } 1-Q<y<Q, \\
\frac{1}{2} \alpha_{3 k-L-1, k} & \text { if } Q<y<Q+1,\end{cases} \\
n_{k, L}= \begin{cases}\max \left\{n_{3 k-L+1, k}+1, N\right\} & \text { if }-Q-1<y<-Q, \\
\max \left\{n_{3 k-L+1, k}+1, n_{3 k-L, k}+1, N\right\} & \text { if }-Q<y<Q-1, \\
\max \left\{n_{3 k-L, k}+1, N\right\} & \text { if } Q-1<y<1-Q, \\
\max \left\{n_{3 k-L-1, k}+1, n_{3 k-L, k}+1, N\right\} & \text { if } 1-Q<y<Q, \\
\max \left\{n_{3 k-L-1, k}+1, N\right\} & \text { if } Q<y<Q+1 .\end{cases}
\end{array}
$$

If $n \geq n_{k, L}$ is an integer and $x \in[0,1-Q]$, then putting

$$
w=y+q^{n-1} x, \quad z=y-q^{n-1} x+q^{n-1},
$$

we have

$$
q w=q^{n} x+q y=q^{n} x+k-L q, \quad q z=-\left(q^{n} x-q^{n}-k+L q\right)
$$


and, in consequence,

$$
\begin{aligned}
& f\left(q^{n} x+k-L q\right)= f(q w)=\frac{1}{4 q}[f(w-1)+f(w+1)+2 f(w)] \\
&= \begin{cases}\frac{1}{4 q} f(w+1) & \text { if }-Q-1<w<-Q, \\
\frac{1}{4 q}[f(w+1)+2 f(w)] & \text { if }-Q<w<Q-1, \\
\frac{1}{2 q} f(w) & \text { if } Q-1<w<1-Q, \\
\frac{1}{4 q}[f(w-1)+2 f(w)] & \text { if } 1-Q<w<Q, \\
\frac{1}{4 q} f(w-1) & \text { if } Q<w<Q+1,\end{cases} \\
&= \begin{cases}\frac{1}{4 q} f(w+1) & \text { if }-Q-1<y<-Q, \\
\frac{1}{4 q}[f(w+1)+2 f(w)] & \text { if }-Q<y<Q-1, \\
\frac{1}{2 q} f(w) & \text { if } Q-1<y<1-Q, \\
\frac{1}{4 q}[f(w-1)+2 f(w)] & \text { if } 1-Q<y<Q, \\
\frac{1}{4 q} f(w-1) & \text { if } Q<y<Q+1,\end{cases} \\
&=\alpha_{k, L}\left(\frac{1}{2 q}\right)^{n} f(x),
\end{aligned}
$$

and

$$
\begin{aligned}
f\left(q^{n} x-q^{n}-k+L q\right)= & f(-q z)=\frac{1}{4 q}[f(-z-1)+f(-z+1)+2 f(-z)] \\
& = \begin{cases}\frac{1}{4 q} f(-z-1) & \text { if }-Q-1<y<-Q, \\
\frac{1}{4 q}[f(-z-1)+2 f(-z)] & \text { if }-Q<y<Q-1, \\
\frac{1}{2 q} f(-z) & \text { if } Q-1<y<1-Q, \\
\frac{1}{4 q}[f(-z+1)+2 f(-z)] & \text { if } 1-Q<y<Q, \\
\frac{1}{4 q} f(-z+1) & \text { if } Q<y<Q+1,\end{cases} \\
= & \frac{1}{2} \alpha_{k, L}\left(\frac{1}{2 q}\right)^{n} f(x) .
\end{aligned}
$$

Theorem 1. If $q=(3-\sqrt{5}) / 2$, then

$$
B_{q}=C_{q}=Z_{q}=[-Q, Q] .
$$

Proof. Assume $f$ is a solution of Schilling's problem which is bounded in a neighbourhood of $x_{0} \in[-Q, Q]$. Since $\mathbb{Z}+q \mathbb{Z}$ is a dense subset of the real line, we may (and do) assume that $x_{0}$ is of the form $k-l q$, where $k$ and $l$ are integers satisfying (6). This jointly with (5) implies that $k \cdot l \geq 0$.

If $x \in[0,1-Q]$, then either the left-hand side of (7) or the left-hand side of (8) is bounded with respect to $n$. From Lemma 4 we then infer that $f$ vanishes on $[0,1-Q]$. Now by the second part of (5) and Lemma 1 it is obvious that $f$ vanishes everywhere. 
Putting $x=0$ in (7) and using Remarks 1 and 2 we get one of the main results of [15].

Corollary 1 . If $q=(3-\sqrt{5}) / 2$, then every solution of Schilling's problem vanishes on $\mathbb{Z}+q \mathbb{Z}$.

In the second part of this paper we will show that in Theorem 1 the number $(3-\sqrt{5}) / 2$ may be replaced by any $q \in(1 / 3,1 / 2)$ satisfying

$$
2 \sum_{k=1}^{K} q^{k}+\lambda q^{K+1}=1
$$

with a positive integer $K$ and a $\lambda \in\{1,2\}$.

Lemma 5. If there exist $K \in \mathbb{N}$ and $\lambda \in\{1,2\}$ satisfying (12), then

$$
1-Q \neq \sum_{n=0}^{N} \varepsilon_{n} q^{n}
$$

for all $N \in \mathbb{N} \cup\{0\}$ and $\varepsilon_{0}, \ldots, \varepsilon_{N} \in\{-1,0,1\}$.

Proof. Suppose that there exist $N \in \mathbb{N} \cup\{0\}$ and $\varepsilon_{0}, \ldots, \varepsilon_{N} \in\{-1,0,1\}$ such that

$$
1-Q=\sum_{n=0}^{N} \varepsilon_{n} q^{n}
$$

and put

$$
x_{0}=\sum_{n=0}^{N} \varepsilon_{n} q^{n} .
$$

We conclude from (12) that

$$
1-Q=2 \sum_{n=1}^{K} q^{n}+\lambda q^{K+1}-\sum_{n=1}^{\infty} q^{n},
$$

hence

$$
x_{0}=\sum_{n=1}^{K} q^{n}+(\lambda-1) q^{K+1}-\sum_{n=K+2}^{\infty} q^{n} .
$$

Moreover, $q<1 / 2$ implies

$$
\sum_{n=K+2}^{\infty} q^{n}<q^{K+1}<q^{K},
$$

which jointly with (15) gives

$$
\sum_{n=1}^{K-1} q^{n}<\sum_{n=1}^{K} q^{n}-\sum_{n=K+2}^{\infty} q^{n} \leq x_{0} .
$$


We are now in a position to show

$$
\varepsilon_{0}=0, \quad N \geq K, \quad \varepsilon_{n}=1 \quad \text { for } n \in\{1, \ldots, K-1\} .
$$

In fact, if $\varepsilon_{0} \neq 0$, then using (13) and (14) we have $x_{0}-\varepsilon_{0}=1-Q-\varepsilon_{0} \in$ $\{-Q, 2-Q\}$ which contradicts $x_{0}-\varepsilon_{0} \in(-Q, Q)$ resulting from (14). Thus $\varepsilon_{0}=0$. Hence (14) yields

$$
x_{0}=\sum_{n=1}^{N} \varepsilon_{n} q^{n} \leq \sum_{n=1}^{N} q^{n} .
$$

By (16) we therefore get $N \geq K$. Suppose now that $\varepsilon_{i} \neq 1$ for some $i \in$ $\{1, \ldots, K-1\}$. Then

$$
x_{0}=\sum_{n=1}^{N} \varepsilon_{n} q^{n} \leq \sum_{n=1}^{i-1} q^{n}+\sum_{n=i+1}^{N} q^{n} \leq \sum_{n=1}^{K-2} q^{n}+\sum_{n=K}^{N} q^{n}<\sum_{n=1}^{K-1} q^{n},
$$

contrary to (16).

From (14) and (17) we have

$$
x_{0}=\sum_{n=1}^{K-1} q^{n}+\sum_{n=K}^{N} \varepsilon_{n} q^{n}
$$

which jointly with (15) gives

$$
\sum_{n=0}^{N-K} \varepsilon_{K+n} q^{n}=1+(\lambda-1) q-\sum_{n=2}^{\infty} q^{n}
$$

Consider first the case $\lambda=2$. Then (18) reads

$$
\sum_{n=0}^{N-K} \varepsilon_{K+n} q^{n}=1+q-\sum_{n=2}^{\infty} q^{n}
$$

If $\varepsilon_{K} \neq 1$, then

$$
\sum_{n=0}^{N-K} \varepsilon_{K+n} q^{n} \leq \sum_{n=1}^{N-K} \varepsilon_{K+n} q^{n}<Q<1<1+q-\sum_{n=2}^{\infty} q^{n} .
$$

This contradicts (19), so $\varepsilon_{K}=1$. Thus (19) leads to

$$
\sum_{n=0}^{N-K-1} \varepsilon_{K+1+n} q^{n}=1-\sum_{n=1}^{\infty} q^{n}=1-Q
$$

i.e.,

$$
1-Q=\sum_{n=0}^{N-K} \varepsilon_{n}^{\prime} q^{n}
$$


where $\varepsilon_{n}^{\prime}=\varepsilon_{K+1+n}$ for $n \in\{0, \ldots, N-K-1\}$ and $\varepsilon_{N-K}^{\prime}=0$. Of course

$$
\varepsilon_{n}^{\prime} \in\{-1,0,1\} \quad \text { for } n \in\{0, \ldots, N-K\} .
$$

Assume now that $\lambda=1$. Then (18) reduces to

$$
\sum_{n=0}^{N-K} \varepsilon_{K+n} q^{n}=1-\sum_{n=2}^{\infty} q^{n} .
$$

In particular, $N \neq K$. This and (17) give $N \geq K+1$.

If $\varepsilon_{K+1} \neq-1$, then $\varepsilon_{K+1}-1 \in\{-1,0\}$ and (22) can be written in the form

$$
\varepsilon_{K}+\left(\varepsilon_{K+1}-1\right) q+\sum_{n=2}^{N-K} \varepsilon_{K+n} q^{n}=1-Q,
$$

i.e., in the form (20), where now $\varepsilon_{0}^{\prime}=\varepsilon_{K}, \varepsilon_{1}^{\prime}=\varepsilon_{K+1}-1$ and $\varepsilon_{n}^{\prime}=\varepsilon_{K+n}$ for $n \in\{2, \ldots, N-K\}$. Moreover, (21) holds as well.

Finally assume that $\varepsilon_{K+1}=-1$. From (22) we have

$$
\varepsilon_{K}-q+\sum_{n=2}^{N-K} \varepsilon_{K+n} q^{n}=1-\sum_{n=2}^{\infty} q^{n}>1-q .
$$

Hence

$$
1<\varepsilon_{K}+\sum_{n=2}^{N-K} \varepsilon_{K+n} q^{n}<\varepsilon_{K}+1
$$

Therefore $\varepsilon_{K}=1$ and equality (22) can also be written in the form

$$
\sum_{n=1}^{N-K-1} \varepsilon_{K+1+n} q^{n}=1-Q .
$$

In each of the cases considered we have represented $1-Q$ in the form (20) with (21). Consequently, we have shown that if $1-Q$ is of the form (13), then $N \geq K$ and $1-Q$ is of the form (20) as well. Consequently, $N \geq m K$ for every positive integer $m$, a contradiction.

Lemma 6. Assume that (12) holds for some $K \in \mathbb{N}$ and $\lambda \in\{1,2\}$. If $N$ is a positive integer, $\varepsilon_{0} \in\{-1,1\}, \varepsilon_{1}, \ldots, \varepsilon_{N} \in\{-1,0,1\}$, and the number $x_{0}$ defined by (14) belongs to $[-Q, Q]$, then $N \geq K$ and $\varepsilon_{n}=-\varepsilon_{0}$ for all $n \in\{1, \ldots, K\}$. Moreover, if $\lambda=2$, then $N \geq K+1$ and $\varepsilon_{K+1} \neq \varepsilon_{0}$.

Proof. Combining (14) with (12) we obtain

$$
\left|x_{0}\right|=\left|\varepsilon_{0}+\sum_{n=1}^{N} \varepsilon_{0} q^{n}\right| \geq 1-\sum_{n=1}^{N} q^{n}=2 \sum_{n=1}^{K} q^{n}+\lambda q^{K+1}-\sum_{n=1}^{N} q^{n} .
$$


Suppose that $N<K$. Then

$$
\left|x_{0}\right| \geq \sum_{n=1}^{K} q^{n}+\sum_{n=N+1}^{K} q^{n}+\lambda q^{K+1}>\sum_{n=1}^{K} q^{n}+q^{K}>Q
$$

a contradiction.

Suppose now that $\varepsilon_{i} \neq-\varepsilon_{0}$ for some $i \in\{1, \ldots, K\}$. Then

$$
\begin{aligned}
\left|x_{0}\right| & =\left|\varepsilon_{0}+\sum_{n=1}^{i-1} \varepsilon_{n} q^{n}+\varepsilon_{i} q^{i}+\sum_{n=i+1}^{N} \varepsilon_{n} q^{n}\right| \\
& \geq 1-\sum_{n=1}^{i-1} q^{n}-\sum_{n=i+1}^{N} q^{n} \\
& =2 \sum_{n=1}^{K} q^{n}+\lambda q^{K+1}-\sum_{n=1, n \neq i}^{N} q^{n} \\
& \geq 2 \sum_{n=1}^{K} q^{n}+\lambda q^{K+1}-\sum_{n=1, n \neq K}^{N} q^{n} \\
& =\sum_{n=1}^{K} q^{n}+\lambda q^{K+1}+q^{K}-\sum_{n=K}^{N} q^{n} \\
& >\sum_{n=1}^{K} q^{n}+q^{K+1}+q^{K}-\sum_{n=K+1}^{\infty} q^{n} \\
& =Q-2 \sum_{n=K+1}^{\infty} q^{n}+q^{K+1}+q^{K} .
\end{aligned}
$$

Moreover, since (12) implies $2 q+q^{2} \leq 1$, we have

$$
2 \sum_{n=K+1}^{\infty} q^{n}=2 \frac{q^{K+1}}{1-q} \leq q^{K}+q^{K+1}
$$

Hence $\left|x_{0}\right|>Q$, which contradicts our assumption.

Assume now $\lambda=2$. If $N=K$, then from (23) we get

$$
\left|x_{0}\right| \geq \sum_{n=1}^{K} q^{n}+2 q^{K+1}=\sum_{n=1}^{K+1} q^{n}+q^{K+1}>Q
$$

a contradiction which shows that $N \geq K+1$. It remains to show that 
$\varepsilon_{K+1} \neq \varepsilon_{0}$. Indeed, if $\varepsilon_{K+1}=\varepsilon_{0}$, then

$$
\begin{aligned}
\left|x_{0}\right|= & \left|\varepsilon_{0}+\sum_{n=1}^{K} \varepsilon_{n} q^{n}+\varepsilon_{0} q^{K+1}+\sum_{n=K+2}^{N} \varepsilon_{n} q^{n}\right| \\
& \geq 1-\sum_{n=1}^{K} q^{n}+q^{K+1}-\sum_{n=K+2}^{N} q^{n} \\
& =2 \sum_{n=1}^{K} q^{n}+2 q^{K+1}-\sum_{n=1}^{K} q^{n}+q^{K+1}-\sum_{n=K+2}^{N} q^{n} \\
& >\sum_{n=1}^{K+1} q^{n}+2 q^{K+1}-\sum_{n=K+2}^{\infty} q^{n} \\
& >\sum_{n=1}^{K+1} q^{n}+q^{K+1}>Q,
\end{aligned}
$$

contrary to the assumption.

LEMMA 7. Assume $q \in(0,1 / 2)$. If $f$ is a solution of Schilling's problem, then

$$
f\left(q^{N+1} y+2 \varepsilon q^{N+1}+\varepsilon \sum_{n=1}^{N} q^{n}\right)=\left(\frac{1}{4 q}\right)^{N+1} f(y+\varepsilon)
$$

for all $N \in \mathbb{N} \cup\{0\}, \varepsilon \in\{-1,1\}$, and $|y| \leq 1$.

Proof. Notice that if $\varepsilon=-1$, then $y+2 \varepsilon \leq-1$, and if $\varepsilon=1$, then $y+2 \varepsilon \geq 1$. This gives

$$
\begin{aligned}
f(q y+2 \varepsilon q) & =\frac{1}{4 q}[f(y+2 \varepsilon-1)+f(y+2 \varepsilon+1)+2 f(y+2 \varepsilon)] \\
& =\frac{1}{4 q} f(y+\varepsilon)
\end{aligned}
$$

and (24) remains true for $N=0$.

Assuming (24) to hold for a nonnegative integer $N$, put

$$
z=q^{N+1} y+2 \varepsilon q^{N+1}+\varepsilon \sum_{n=1}^{N} q^{n}
$$

and observe that if $\varepsilon=-1$, then

$$
z=q^{N+1} y-2 q^{N+1}-\sum_{n=1}^{N} q^{n} \leq-\sum_{n=1}^{N+1} q^{n}<0<1-Q,
$$

while if $\varepsilon=1$, then 


$$
z=q^{N+1} y+2 q^{N+1}+\sum_{n=1}^{N} q^{n} \geq \sum_{n=1}^{N+1} q^{n}>0>Q-1 .
$$

It follows that

$$
\begin{aligned}
f\left(q^{N+2} y\right. & \left.+2 \varepsilon q^{N+2}+\varepsilon \sum_{n=1}^{N+1} q^{n}\right)=f(q z+q \varepsilon) \\
& =\frac{1}{4 q}[f(z+\varepsilon-1)+f(z+\varepsilon+1)+f(z+\varepsilon)]=\frac{1}{4 q} f(z) \\
& =\frac{1}{4 q} f\left(q^{N+1} y+2 \varepsilon q^{N+1}+\varepsilon \sum_{n=1}^{N} q^{n}\right)=\left(\frac{1}{4 q}\right)^{N+2} f(y+\varepsilon) .
\end{aligned}
$$

LEMma 8. Assume that (12) holds for some $K \in \mathbb{N}$ and $\lambda \in\{1,2\}$, $n$ is a nonnegative integer, $\varepsilon_{0}, \ldots, \varepsilon_{N} \in\{-1,0,1\}$ and the number $x_{0}$ defined by (14) belongs to $[-Q, Q]$. If $f$ is a solution of Schilling's problem, then there exist $\alpha_{\varepsilon_{0}, \ldots, \varepsilon_{N}}>0$ and a positive integer $n_{\varepsilon_{0}, \ldots, \varepsilon_{N}}$ such that

$$
f\left(q^{N+n} x+x_{0}\right)=\alpha_{\varepsilon_{0}, \ldots, \varepsilon_{N}}\left(\frac{1}{2 q}\right)^{N+n} f(x)
$$

for every integer $n \geq n_{\varepsilon_{0}, \ldots, \varepsilon_{N}}$ and $x \in[Q-1,1-Q]$.

Proof. If $N=0$, then $\left|\varepsilon_{0}\right|=\left|x_{0}\right| \leq Q<1$. Consequently, $\varepsilon_{0}=0$ and $x_{0}=0$. Hence for $N=0$ it is enough to put $\alpha_{\varepsilon_{0}}=1, n_{\varepsilon_{0}}=1$ and use Lemma 2.

Fix now a nonnegative integer $M$ and assume that for every nonnegative integer $N \leq M$ and every $\varepsilon_{0}, \ldots, \varepsilon_{N} \in\{-1,0,1\}$ such that $x_{0}$ belongs to $[-Q, Q]$ there exist $\alpha_{\varepsilon_{0}, \ldots, \varepsilon_{N}}>0$ and a positive integer $n_{\varepsilon_{0}, \ldots, \varepsilon_{N}}$ such that (25) holds for all $n \geq n_{\varepsilon_{0}, \ldots, \varepsilon_{N}}$ and $x \in[Q-1,1-Q]$.

Fix $\varepsilon_{0}, \ldots, \varepsilon_{M+1} \in\{-1,0,1\}$ and assume that

$$
y_{0}=\sum_{m=0}^{M+1} \varepsilon_{m} q^{m} \in[-Q, Q] \text {. }
$$

Consider the following three cases:

(i) $\varepsilon_{0}=0$,

(ii) $\left|\varepsilon_{0}\right|=1$ and $\lambda=1$,

(iii) $\left|\varepsilon_{0}\right|=1$ and $\lambda=2$.

In case (i) from Lemma 5 we see that $y=q^{-1} y_{0}$ belongs to one of the intervals (10). Then there exists a positive integer $L$ such that for every $n \geq L$ and every $x \in[Q-1,1-Q]$ the number $y$ belongs to one of the intervals (10) together with $q^{M+n} x+y$. Observe also that if $y<Q-1$, then $\varepsilon_{1} \neq 1$, and if $y>1-Q$, then $\varepsilon_{1} \neq-1$. In particular, we can define 


$$
\begin{aligned}
\alpha_{\varepsilon_{0}, \ldots, \varepsilon_{M+1}}= \begin{cases}\frac{1}{2} \alpha_{\varepsilon_{1}+1, \varepsilon_{2}, \ldots, \varepsilon_{M+1}} & \text { if }-Q-1<y<-Q, \\
\frac{1}{2}\left[\alpha_{\varepsilon_{1}+1, \varepsilon_{2}, \ldots, \varepsilon_{M+1}}+2 \alpha_{\varepsilon_{1}, \varepsilon_{2}, \ldots, \varepsilon_{M+1}}\right] & \text { if }-Q<y<Q-1, \\
\alpha_{\varepsilon_{1}, \ldots, \varepsilon_{M+1}} & \text { if } Q-1<y<1-Q, \\
\frac{1}{2}\left[\alpha_{\varepsilon_{1}-1, \varepsilon_{2}, \ldots, \varepsilon_{M+1}}+2 \alpha_{\left.\varepsilon_{1}, \varepsilon_{2}, \ldots, \varepsilon_{M+1}\right]}\right] & \text { if } 1-Q<y<Q, \\
\frac{1}{2} \alpha_{\varepsilon_{1}-1, \varepsilon_{2}, \ldots, \varepsilon_{M+1}} & \text { if } Q<y<Q+1,\end{cases} \\
n_{\varepsilon_{0}, \ldots, \varepsilon_{M+1}}= \begin{cases}\max \left\{n_{\varepsilon_{1}+1, \varepsilon_{2}, \ldots, \varepsilon_{M+1}}, L\right\} & \text { if }-Q-1<y<-Q, \\
\max \left\{n_{\varepsilon_{1}+1, \varepsilon_{2}, \ldots, \varepsilon_{M+1}}, n_{\varepsilon_{1}, \ldots, \varepsilon_{M+1}}, L\right\} & \text { if }-Q<y<Q-1, \\
\max \left\{n_{\varepsilon_{1}, \ldots, \varepsilon_{M+1}}, L\right\} & \text { if } Q-1<y<1-Q, \\
\max \left\{n_{\varepsilon_{1}-1, \varepsilon_{2}, \ldots, \varepsilon_{M+1}}, n_{\varepsilon_{1}, \ldots, \varepsilon_{M+1}}, L\right\} & \text { if } 1-Q<y<Q \\
\max \left\{n_{\varepsilon_{1}-1, \varepsilon_{2}, \ldots, \varepsilon_{M+1}}, L\right\} & \text { if } Q<y<Q+1 .\end{cases}
\end{aligned}
$$

If $n \geq n_{\varepsilon_{0}, \ldots, \varepsilon_{M+1}}$ and $x \in[Q-1,1-Q]$, then putting $w=q^{M+n} x+y$ we have

$$
\begin{aligned}
f\left(q^{M+1+n} x+y_{0}\right)= & f(q w)=\frac{1}{4 q}[f(w-1)+f(w+1)+2 f(w)] \\
& = \begin{cases}\frac{1}{4 q} f(w+1) & \text { if }-Q-1<y<-Q, \\
\frac{1}{4 q}[f(w+1)+2 f(w)] & \text { if }-Q<y<Q-1, \\
\frac{1}{2 q} f(w) & \text { if } Q-1<y<1-Q, \\
\frac{1}{4 q}[f(w-1)+2 f(w)] & \text { if } 1-Q<y<Q, \\
\frac{1}{4 q} f(w-1) & \text { if } Q<y<Q+1, \\
& =\alpha_{\varepsilon_{0}, \ldots, \varepsilon_{M+1}\left(\frac{1}{2 q}\right)^{M+1+n} f(x) .}\end{cases}
\end{aligned}
$$

Consider now case (ii). According to Lemma $6, M+1 \geq K$, and $\varepsilon_{m}=-\varepsilon_{0}$ for $m \in\{1, \ldots, K\}$. Applying now (12) we see that

$$
y_{0}=\varepsilon_{0}-\sum_{m=1}^{K} \varepsilon_{0} q^{m}+\sum_{m=K+1}^{M+1} \varepsilon_{m} q^{m}=\sum_{m=1}^{K+1} \varepsilon_{0} q^{m}+\sum_{m=K+1}^{M+1} \varepsilon_{m} q^{m}
$$

Put

$$
\begin{aligned}
& \alpha_{\varepsilon_{0}, \ldots, \varepsilon_{M+1}}=\left\{\begin{array}{lr}
\left(\frac{1}{2}\right)^{K+1} & \text { if } M+1=K, \\
\alpha_{0, \varepsilon_{0}, \ldots, \varepsilon_{0}}, \varepsilon_{K+1}+\varepsilon_{0}, \varepsilon_{K+2}, \ldots, \varepsilon_{M+1} & \text { if } M+1>K, \\
\underbrace{}_{K} & \varepsilon_{K+1} \in\left\{0,-\varepsilon_{0}\right\}, \\
\left(\frac{1}{2}\right)^{K+1} \alpha_{\varepsilon_{0}, \varepsilon_{K+2}, \ldots, \varepsilon_{M+1}} & \text { if } M+1>K, \varepsilon_{K+1}=\varepsilon_{0},
\end{array}\right. \\
& n_{\varepsilon_{0}, \ldots, \varepsilon_{M+1}}= \begin{cases}1 & \text { if } M+1=K, \\
n_{0,} \underbrace{\varepsilon_{0}, \ldots, \varepsilon_{0}}_{K}, \varepsilon_{K+1}+\varepsilon_{0}, \varepsilon_{K+2}, \ldots, \varepsilon_{M+1} & \text { if } M+1>K, \\
n_{\varepsilon_{0}, \varepsilon_{K+2}, \ldots, \varepsilon_{M+1}} & \varepsilon_{K+1} \in\left\{0,-\varepsilon_{0}\right\},\end{cases} \\
& \text { if } M+1>K, \varepsilon_{K+1}=\varepsilon_{0},
\end{aligned}
$$

and fix $n \geq n_{\varepsilon_{0}, \ldots, \varepsilon_{M+1}}$ and $x \in[Q-1,1-Q]$. 
If $M+1=K$, then by (26) and Lemma 2 we get

$$
\begin{aligned}
f\left(q^{M+1+n} x+y_{0}\right) & =f\left(q^{K+n} x+\varepsilon_{0} \sum_{m=1}^{K+1} q^{m}\right)=\left(\frac{1}{2}\right)^{K+1}\left(\frac{1}{2 q}\right)^{K+n} f(x) \\
& =\alpha_{\varepsilon_{0}, \ldots, \varepsilon_{M+1}}\left(\frac{1}{2 q}\right)^{M+1+n} f(x) .
\end{aligned}
$$

If $M+1>K$ and $\varepsilon_{K+1} \in\left\{0,-\varepsilon_{0}\right\}$, then by (26) and the proof of case (i) we have

$$
\begin{aligned}
f\left(q^{M+1+n} x+y_{0}\right) & =\alpha_{0, \varepsilon_{0}, \ldots, \varepsilon_{0}, \varepsilon_{K+1}+\varepsilon_{0}, \varepsilon_{K+2}, \ldots, \varepsilon_{M+1}}\left(\frac{1}{2 q}\right)^{M+1+n} f(x) \\
& =\alpha_{\varepsilon_{0}, \ldots, \varepsilon_{M+1}}\left(\frac{1}{2 q}\right)^{M+1+n} f(x) .
\end{aligned}
$$

If $M+1>K$ and $\varepsilon_{K+1}=\varepsilon_{0}$, then (26) takes the form

$$
y_{0}=\sum_{m=1}^{K} \varepsilon_{0} q^{m}+2 \varepsilon_{0} q^{K+1}+\sum_{m=K+2}^{M+1} \varepsilon_{m} q^{m},
$$

and, since $y_{0} \in[-Q, Q]$ and $Q=q^{K+1}(Q+1)+\sum_{m=1}^{K} q^{m}$, we have

$$
2 \varepsilon_{0}+\sum_{m=1}^{M-K} \varepsilon_{K+1+m} q^{m} \in[-Q-1, Q+1] .
$$

Hence, because $\left|\varepsilon_{0}\right|=1$ and the remaining $\varepsilon$ 's are from $\{-1,0,1\}$, we get

$$
\varepsilon_{0}+\sum_{m=1}^{M-K} \varepsilon_{K+1+m} q^{m} \in[-Q, Q] .
$$

Applying (27), Lemma 7 and the induction hypothesis for $x \in[Q-1,1-Q]$ we obtain

$$
\begin{aligned}
f\left(q^{M+1+n} x+y_{0}\right)= & f\left(q^{K+1}\left(q^{M-K+n} x+\sum_{m=1}^{M-K} \varepsilon_{K+1+m} q^{m}\right)\right. \\
& \left.+2 \varepsilon_{0} q^{K+1}+\varepsilon_{0} \sum_{m=1}^{K} q^{m}\right) \\
= & \left(\frac{1}{4 q}\right)^{K+1} f\left(q^{M-K+n} x+\sum_{m=1}^{M-K} \varepsilon_{K+1+m} q^{m}+\varepsilon_{0}\right) \\
= & \left(\frac{1}{4 q}\right)^{K+1} \alpha_{\varepsilon_{0}, \varepsilon_{K+2}, \ldots, \varepsilon_{M+1}}\left(\frac{1}{2 q}\right)^{M-K+n} f(x) \\
= & \alpha_{\varepsilon_{0}, \ldots, \varepsilon_{M+1}}\left(\frac{1}{2 q}\right)^{M+1+n} f(x) .
\end{aligned}
$$


Finally assume (iii) holds. Now Lemma 6 says that $M+1 \geq K+1$, $\varepsilon_{m}=-\varepsilon_{0}$ for all $m \in\{1, \ldots, K\}$ and $\varepsilon_{K+1} \in\left\{0,-\varepsilon_{0}\right\}$. Applying (12) again, we obtain

$$
\begin{aligned}
y_{0} & =\varepsilon_{0}-\sum_{m=1}^{K} \varepsilon_{0} q^{m}+\varepsilon_{K+1} q^{K+1}+\sum_{m=K+2}^{M+1} \varepsilon_{m} q^{m} \\
& =\sum_{m=1}^{K} \varepsilon_{0} q^{m}+\left(2 \varepsilon_{0}+\varepsilon_{K+1}\right) q^{K+1}+\sum_{m=K+2}^{M+1} \varepsilon_{m} q^{m} .
\end{aligned}
$$

Put

$$
\begin{aligned}
& \alpha_{\varepsilon_{0}, \ldots, \varepsilon_{M+1}}= \begin{cases}\left(\frac{1}{2}\right)^{K+1} \alpha_{\varepsilon_{0}, \varepsilon_{K+2}, \ldots, \varepsilon_{M+1}} & \text { if } \varepsilon_{K+1}=0, \\
\alpha_{0,} \underbrace{\varepsilon_{0}, \ldots, \varepsilon_{0}}_{K+1}, \varepsilon_{K+2}, \ldots, \varepsilon_{M+1} & \text { if } \varepsilon_{K+1}=-\varepsilon_{0},\end{cases} \\
& n_{\varepsilon_{0}, \ldots, \varepsilon_{M+1}}= \begin{cases}n_{\varepsilon_{0}, \varepsilon_{K+2}, \ldots, \varepsilon_{M+1}} & \text { if } \varepsilon_{K+1}=0, \\
n_{0, \varepsilon_{K+1}^{\varepsilon_{0}, \ldots, \varepsilon_{0}}, \varepsilon_{K+2}, \ldots, \varepsilon_{M+1}} & \text { if } \varepsilon_{K+1}=-\varepsilon_{0},\end{cases}
\end{aligned}
$$

and fix $n \geq n_{\varepsilon_{0}, \ldots, \varepsilon_{M+1}}$ and $x \in[Q-1,1-Q]$.

If $\varepsilon_{K+1}=0$, then (29) implies (27) and hence also (28).

If $\varepsilon_{K+1}=-\varepsilon_{0}$, then using (29) and part (i) we get

$$
\begin{aligned}
f\left(q^{M+1+n} x+y_{0}\right) & =\alpha_{0, \varepsilon_{0}, \ldots, \varepsilon_{0}, \varepsilon_{K+2}, \ldots, \varepsilon_{M+1}}\left(\frac{1}{2 q}\right)^{M+1+n} f(x) \\
& =\alpha_{\varepsilon_{0}, \ldots, \varepsilon_{M+1}}\left(\frac{1}{2 q}\right)^{M+1+n} f(x) .
\end{aligned}
$$

Theorem 2. If there exist $K \in \mathbb{N}$ and $\lambda \in\{1,2\}$ satisfying (12), then (11) holds.

Proof. Assume $f$ is a solution of Schilling's problem which is bounded in a neighbourhood of $x_{0} \in[-Q, Q]$. Since

$$
\left\{\sum_{n=0}^{N} \varepsilon_{n} q^{n}: \varepsilon_{0}, \ldots, \varepsilon_{N} \in\{-1,0,1\}, N \in \mathbb{N}\right\}
$$

is dense in $[-Q, Q]$, applying Lemma 8 and arguing as in Theorem 1 we see that $f$ vanishes on $[Q-1,1-Q]$. We will show that $f$ vanishes on $[0, q)$, which jointly with Lemma 1 will complete the proof.

From (12) we get $2 q+q^{2} \leq 1$, so $q^{-1}(1-Q) \geq Q$. If $x \in(1-Q, q)$, then $Q \leq q^{-1}(1-Q)<q^{-1} x$ and $Q-1<q^{-1} x-1<0<1-Q$, whence

$$
f(x)=\frac{1}{4 q}\left[f\left(q^{-1} x-1\right)+f\left(q^{-1} x+1\right)+f\left(q^{-1} x\right)\right]=0 .
$$


We conclude with the following simple consequence of Lemma 8 and Remark 1.

Corollary 2. If there exist $K \in \mathbb{N}$ and $\lambda \in\{1,2\}$ satisfying (12), then every solution of Schilling's problem vanishes on the set (30).

Acknowledgments. This research was supported by the State Committee for Scientific Research Grant No. 2 P03A 03311.

\section{References}

[1] K. Baron, On a problem of R. Schilling, Ber. Math.-Statist. Sekt. Forsch. Graz 286 (1988).

[2] K. Baron and W. Jarczyk, Recent results on functional equations in a single variable, perspectives and open problems, Aequationes Math. 61 (2001), 1-48.

[3] K. Baron, A. Simon et P. Volkmann, Solutions d'une équation fonctionnelle dans l'espace des distributions tempérées, C. R. Acad. Sci. Paris Sér. I Math. 319 (1994), $1249-1252$.

[4] K. Baron et P. Volkmann, Unicité pour une équation fonctionnelle, Wyż. Szkoła Ped. Kraków Rocznik Nauk.-Dydakt. Prace Mat. 13 (1993), 53-56.

[5] L. Bartłomiejczyk, Solutions with big graph of homogeneous functional equations in a single variable, Aequationes Math. 56 (1998), 149-156.

[6] M. J. Bertin, A. Decomps-Guilloux, M. Grandet-Hugot, M. Pathiaux-Delefosse and J. P. Schreiber, Pisot and Salem Numbers, Birkhäuser, 1992.

[7] J. M. Borwein and R. Girgensohn, Functional equations and distribution functions, Results Math. 26 (1994), 229-237.

[8] I. Daubechies and J. C. Lagarias, Two-scale difference equations; I. Existence and global regularity of solutions, SIAM J. Math. Anal. 22 (1991), 1388-1410.

[9] G. Derfel and R. Schilling, Spatially chaotic configurations and functional equations with rescaling, J. Phys. A 29 (1996), 4537-4547.

[10] W. Förg-Rob, On a problem of R. Schilling, Math. Pannon. 5 (1994); I: 29-65, II: $145-168$.

[11] R. Girgensohn, A survey of results and open problems on the Schilling equation, manuscript.

[12] R. Girgensohn and J. Morawiec, Positivity of Schilling functions, Bull. Polish Acad. Sci. Math. 48 (2000), 407-412.

[13] J. Morawiec, Bounded solutions of Schilling's problem, Math. Pannon. 7 (1996), 223-232.

[14] —, On bounded solutions of a problem of R. Schilling, Ann. Math. Sil. 8 (1994), 97-101.

[15] - On continuous solutions of a problem of R. Schilling, Results Math. 27 (1995), 381-386.

[16] Y. Peres and B. Solomyak, Self-similar measures and intersections of Cantor sets, Trans. Amer. Math. Soc. 350 (1998), 81-90.

[17] Report of meeting. Second special session: Schilling's Problem, The Thirty-fifth International Symposium on Functional Equations (Graz-Mariatrost, 1997), Aequationes Math. 55 (1998), 281-318.

[18] R. Schilling, Spatially chaotic structures, in: H. Thomas (ed.), Nonlinear Dynamics in Solids, Springer, Berlin, 1992, 213-241. 
[19] A. Simon et P. Volkmann, Croissance d'une suite définie par une certaine solution distribution d'une équation fonctionnelle, Seminar LV, No. 2, http://www.mathematik.uni-karlsruhe.de/ ${ }^{\text {semlv. }}$

Institute of Mathematics

Silesian University

Bankowa 14

40-007 Katowice, Poland

E-mail: morawiec@gate.math.us.edu.pl

Reçu par la Rédaction le 13.\%.1998

Révisé le 25.1.2001 